International Mathematical Forum, Vol. 8, 2013, no. 18, 883 - 888 HIKARI Ltd, www.m-hikari.com

\title{
On the Cofiniteness of Generalized Local Cohomology Modules
}

\author{
Sh. Payrovi and I. Khalili Gorji \\ Imam Khomeini International University \\ Postal Code: 34149-1-6818, Qazvin, Iran
}

Copyright (c) 2013 Sh. Payrovi and I. Khalili Gorji. This is an open access article distributed under the Creative Commons Attribution License, which permits unrestricted use, distribution, and reproduction in any medium, provided the original work is properly cited.

\begin{abstract}
Let $R$ be a Noetherian ring, $I$ an ideal of $R$, let $M$ be a finitely generated $R$-module and $N$ a minimax $R$-module. In this paper we show that the following conditions are equivalent : $(i) H_{I}^{0}(M, N)$ is $I$ cofinite; $(i i)$ for any minimax submodule $K$ of $H_{I}^{i}(M, N)$ the $R$-module $\operatorname{Hom}_{R}\left(R / I, H_{I}^{t}(M, N) / K\right)$ is finitely generated, whenever the modules $H_{I}^{0}(M, N), H_{I}^{1}(M, N), \cdots, H_{I}^{t-1}(M, N)$ are minimax. As a consequence it follows that the associated primes of $H_{I}^{t}(M, N) / K$ are finite. This generalize the main result of Yassemi, Khatami and Sharif (2002) and Saremi (2009).
\end{abstract}

Mathematics Subject Classification: 13D45; 14B15, 13E05

Keywords: Generalized local cohomology, Cofinite and Minimax modules

\section{Introduction}

Let $R$ be a commutative Noetherian ring, $I$ an ideal of $R$ and let $X, Y$ be two $R$-modules. We use $H_{I}^{i}(X, Y)$ to denote, for $i \geq 0$, the $i^{\text {th }}$ generalized local cohomology module of $X$ and $Y$ with respect to $I$.

The local cohomology theory has been an significant tool in commutative Algebra and Algebraic Geometry. As a generalization of the ordinary local cohomology modules, Herzog (1974), introduced the generalized local cohomology modules and studied further by Suzuki (1978) and Yassemi (1994). It 
is clear that $H_{I}^{i}(R, Y)$ is just the ordinary local cohomology module $H_{I}^{i}(Y)$ of $Y$ with respect to $I$.

One of the important problems in commutative algebra is determining when the set of associated primes of the $i^{\text {th }}$ local cohomology modules is finite. The purpose of this paper is to extend the main results of Brodmann and Lashgary Faghani (2000), Asadollahi, Khashyarmanesh and Salarian (2002), Yassemi, Khatami and Sharif (2002), Bahmanpour and Naghipour (2008), Saremi (2009) and Borna, Sahandi and Yassemi (2011) to the class of minimax modules.

\section{Main Results}

Definition 2.1 An $R$-module $X$ is said to be zero-divisor module if for any submodule $Y$ of $X$, the set $Z_{R}(X / Y)$ is a finite union of prime ideals in $\operatorname{Ass}_{R}(X / Y)$.

According to Divaani-Aazar and Esmkhani (2005, Example 2.2), the class of zero-divisor modules ( $Z D$-modules) is much larger than that of finitely generated modules.

Definition 2.2 An R-module $X$ is said to be minimax module, if there is a finitely generated submodule $Y$ of $X$ such that $X / Y$ is Artinian.

The class of minimax modules thus includes all finitely generated and all Artinian modules. Moreover, it is a Serre subcategory of the category of $R$ modules and is a subset of the class of $Z D$-modules. Zöschinger $(1986,1988)$ has given many equivalent conditions for a module to be a minimax module.

Definition 2.3 An $R$-module $X$ is said to be $I$-cofinite, whenever $\operatorname{Supp}(X)$ $\subseteq V(I)$ and $\operatorname{Ext}_{R}^{i}(R / I, X)$ is finitely generated $R$-module, for all $i \geq 0$.

In the rest of the paper let $R$ be a commutative Noetherian ring, $I$ an ideal of $R$, let $M$ be a finitely generated $R$-module, $N$ a minimax module and let $t$ be a non-negative integer. We start with the following lemma.

Lemma 2.4 The following statement are equivalent:

(i) $H_{I}^{i}\left(M, \Gamma_{I}(N)\right)$ is I-cofinite, for all $i \leq t$;

(ii) If $H_{I}^{i}(M, N)$ is $I$-cofinite and minimax, for all $i<t$, then the $R$-module $H_{R}\left(R / I, H_{I}^{t}(M, N)\right)$ is finitely generated. 
Proof. The proof of $(i \Rightarrow i i)$, which we include for the reader's convenience, is based on Bahmanpour and Naghipour (2008 Lemma 2.2). We use induction on $t$. When $t=0$, there is nothing to prove, because $H_{I}^{0}(M, N)=$ $\Gamma_{I}\left(\operatorname{Hom}_{R}(M, N)\right)=\operatorname{Hom}_{R}\left(M, \Gamma_{I}(N)\right)=H_{I}^{0}\left(M, \Gamma_{I}(N)\right)$ is I-cofinite, by using Yassemi et al. (2002 Lemma 1.1). Thus $\operatorname{Hom}_{R}\left(R / I, H_{I}^{0}(M, N)\right)$ is finitely generated. Assume, inductively, that $t>0$ and that the result has been proved for smaller values of $t$. By the inductive hypothesis $H_{I}^{i}(M, N)$ is $I$-cofinite and minimax, for all $i<t$, and $H_{I}^{i}\left(M, \Gamma_{I}(N)\right)$ is $I$-cofinite, for all $i \leq t$. It only remain to prove $\operatorname{Hom}_{R}\left(R / I, H_{I}^{t}(M, N)\right)$ is finitely generated. Let $L=N / \Gamma_{I}(N)$. Then the exact sequence $(*) 0 \longrightarrow \Gamma_{I}(N) \longrightarrow N \longrightarrow L \longrightarrow 0$ induces a long exact sequence

$$
\left.\longrightarrow H_{I}^{i}\left(M, \Gamma_{I}(N)\right) \longrightarrow H_{I}^{i}(M, N)\right) \longrightarrow H_{I}^{i}(M, L) \longrightarrow H_{I}^{i+1}\left(M, \Gamma_{I}(N)\right) \longrightarrow
$$

In view of Bahmanpour and Naghipour (2008 Lemma 2.1(i)) any submodule of a minimax module is minimax, also $H_{I}^{i}\left(M, \Gamma_{I}(N)\right)=\operatorname{Ext} t_{R}^{i}\left(M, \Gamma_{I}(N)\right)$ by using Yassemi et al. (2002 Lemma 1.1). Hence, $H_{I}^{i}\left(M, \Gamma_{I}(N)\right)$ is minimax by Saremi (2009 Lemma 2.1(ii)). Now, $H_{I}^{i+1}\left(M, \Gamma_{I}(N)\right)$ and $H_{I}^{i}(M, N)$ are $I$-cofinite and minimax, for all $i<t$, therefore $H_{I}^{i}(M, L)$ is $I$-cofinite and minimax, for all $i<t$, by using Melkersson (2005 Corollary 4.4). We now use Divaani-Aazar and Esmkhani (2005 Lemma 2.4) to deduce that $I$ contain an element $x$ which is a non-zero divisor on $L$. The exact sequence $0 \longrightarrow L \stackrel{x}{\longrightarrow} L \longrightarrow L / x L \longrightarrow 0$ induces the long exact sequence $(* *)$

$$
H_{I}^{i-1}(M, L) \stackrel{x}{\longrightarrow} H_{I}^{i-1}(M, L) \longrightarrow H_{I}^{i-1}(M, L / x L) \longrightarrow H_{I}^{i}(M, L) \stackrel{x}{\longrightarrow}
$$

for each $i \geq 1$. Thus the sequence

$$
0 \longrightarrow H_{I}^{i-1}(M, L) / x H_{I}^{i-1}(M, L) \longrightarrow H_{I}^{i-1}(M, L / x L) \longrightarrow 0:_{H_{I}^{i}(M, L)} x \longrightarrow 0
$$

is exact, for each $i \geq 1$ Therefore, by Melkersson (2005 Corollary 4.4) we have $H_{I}^{i}(M, L / x L)$ is $I$-cofinite and minimax, for all $i<t-1$. Hence, by the inductive hypothesis, $\operatorname{Hom}_{R}\left(R / I, H_{I}^{t-1}(M, L / x L)\right)$ is finitely generated. On the other hand, $\operatorname{Ext}_{R}^{1}\left(R / I, H_{I}^{t-1}(M, L) / x H_{I}^{t-1}(M, L)\right)$ is a finitely generated $R$-module since $H_{I}^{t-1}(M, L) / x H_{I}^{t-1}(M, L)$ is $I$-cofinite. Hence, the exact sequence

$$
\begin{aligned}
\operatorname{Hom}_{R}\left(R / I, H_{I}^{t-1}(M, L / x L)\right) & \longrightarrow \operatorname{Hom}_{R}\left(R / I, 0:_{H_{I}^{t}(M, L)} x\right) \\
& \longrightarrow \operatorname{Ext}_{R}^{1}\left(R / I, H_{I}^{t-1}(M, L) / x H_{I}^{t-1}(M, L)\right)
\end{aligned}
$$

shows that

$$
\begin{aligned}
\operatorname{Hom}_{R}\left(R / I, 0:_{H_{I}^{t}(M, L)} x\right) & \cong \operatorname{Hom}_{R}\left(R / I \otimes R / x R, H_{I}^{t}(M, L)\right) \\
& \cong \operatorname{Hom}_{R}\left(R / I, H_{I}^{t}(M, L)\right)
\end{aligned}
$$


is finitely generated. By using the exact sequence $\left(^{*}\right)$ we have the exact sequence $H_{I}^{t}\left(M, \Gamma_{I}(N)\right) \stackrel{\alpha}{\longrightarrow} H_{I}^{t}(M, N) \stackrel{\beta}{\longrightarrow} H_{I}^{t}(M, L)$. So the sequence $(* * *)$

$0 \longrightarrow \operatorname{Hom}_{R}(R / I, \operatorname{Ker} \beta) \longrightarrow \operatorname{Hom}_{R}\left(R / I, H_{I}^{t}(M, N)\right) \longrightarrow \operatorname{Hom}_{R}(R / I, \operatorname{Im} \beta)$

is exact. We have $\operatorname{Hom}_{R}(R / I, \operatorname{Ker} \beta)$ is finitely generated, since $H_{I}^{t}\left(M, \Gamma_{I}(L)\right)$ $/ K e r \alpha \cong K e r \beta$ is $I$-cofinite. On the other hand, the exact sequence $0 \longrightarrow$ $\operatorname{Im} \beta \longrightarrow H_{I}^{t}(M, L)$ induces the exact sequence $0 \longrightarrow H_{R}(R / I, \operatorname{Im} \beta) \longrightarrow$ $\operatorname{Hom}_{R}\left(R / I, H_{I}^{t}(M, L)\right)$ which shows that $H_{R}(R / I, I m \beta)$ is finitely generated, since $\operatorname{Hom}_{R}\left(R / I, H_{I}^{t}(M, L)\right)$ is finitely generated. Hence, by the exact sequence $\left.{ }^{* * *}\right)$ we have $\operatorname{Hom}_{R}\left(R / I, H_{I}^{t}(M, N)\right)$ is finitely generated. This complete the inductive step. The result has been proved in this case.

$(i i \Rightarrow i)$ We use induction on $t$. For all $i<t=0, H_{I}^{i}(M, N)=0$ is $I$-cofinite and minimax thus by assumption

$$
\operatorname{Hom}_{R}\left(R / I, H_{I}^{0}(M, N)\right)=\operatorname{Hom}_{R}\left(R / I, H_{I}^{0}\left(M, \Gamma_{I}(N)\right)\right)
$$

is finitely generated and therefore $H_{I}^{0}\left(M, \Gamma_{I}(N)\right)$ is $I$-cofinite by Melkersson (2005 Proposition 4.3). Assume, inductively, that $t>0$ and $H_{I}^{i}\left(M, \Gamma_{I}(N)\right)$ is $I$-cofinite for $i=0,1, \cdots, t-1$. Then $\operatorname{Hom}_{R}\left(R / I, H_{R}^{t}\left(M, \Gamma_{I}(N)\right)\right)$ is finitely generated by assumption and so it is $I$-cofinite.

Lemma 2.5 The following statement are equivalent:

(i) $H_{I}^{i}\left(M, \Gamma_{I}(N)\right)$ is I-cofinite, for all $i \leq t$;

(ii) If $H_{I}^{i}(M, N)$ is minimax, for all $i<t$, then the $R$-module $\operatorname{Hom}_{R}\left(R / I, H_{I}^{t}(M, N)\right)$ is finitely generated.

Proof. In view of 2.4 it is enough to show that $H_{I}^{i}(M, N)$ is $I$-cofinite for all $i<t$. We proceed by induction on $i$. The case $i=0$ is obvious as $H_{I}^{0}(M, N)=$ $H_{I}^{0}\left(M, \Gamma_{I}(N)\right)$. So suppose that $i>0$ and that the result has been proved for smaller values of $i$. By the inductive hypothesis $H_{I}^{j}(M, N)$ is $I$-cofinite for $j=0,1, \cdots, i-1$. Therefore, by assumption $\operatorname{Hom}_{R}\left(R / I, H_{I}^{i}(M, N)\right)$ is finitely generated. Hence, $\left.H_{I}^{i}(M, N)\right)$ is $I$-cofinite, by Melkersson (2005 Proposition 4.3). So that $H_{I}^{i}(M, N)$ is $I$-cofinite and minimax, for all $i<t$.

The following result will be used for the generalization of the main result of Yassemi et al. (2002), Saremi (2009) and Borna et al. (2011).

Theorem 2.6 The following statement are equivalent:

(i) $H_{I}^{0}\left(M, \Gamma_{I}(N)\right)$ is I-cofinite;

(ii) If $H_{I}^{i}(M, N)$ is minimax, for all $i<t$, then the $R$-module $H_{R}\left(R / I, H_{I}^{t}(M, N)\right)$ is finitely generated, and so the associated prime ideals of $H_{I}^{t}(M, N)$ is a finite set. 
Proof. By 2.5 it is enough for us to prove that $H_{I}^{i}\left(M, \Gamma_{I}(N)\right)$ is $I$-cofinite for $i \leq t$. We proceed by induction on $i$. The case $i=0$ is obvious by assumption. So suppose that $i>0$ and that the result has been proved for smaller values of $i$. By the inductive hypothesis $H_{I}^{j}\left(M, \Gamma_{I}(N)\right)$ is $I$-cofinite for $j=0,1, \cdots, i-1$. Therefore, by assumption and $2.5 \operatorname{Hom}_{R}\left(R / I, H_{I}^{i}\left(M, \Gamma_{I}(N)\right)\right.$ is finitely generated. Hence, $H_{I}^{i}\left(M, \Gamma_{I}(N)\right)$ is $I$-cofinite, by Melkersson (2005 Proposition 4.3). Therefore, $H_{I}^{i}\left(M, \Gamma_{I}(N)\right)$ is $I$-cofinite, for all $i \leq t$.

Corollary 2.7 Let $\operatorname{obj}(N)$ (resp. obj $(A))$ denote the category of all Noetherian (resp. Artinian) $R$-modules and $R$-homomorphisms. Let $H_{R}^{0}(M, N)$ is $I$-cofinite and $H_{I}^{i}(M, N) \in \operatorname{obj}(N) \cup \operatorname{obj}(A)$, for all $i<t$. Then the $R$-module $H_{\text {om }}\left(R / I, H_{I}^{t}(M, N)\right)$ is finitely generated. In particular, Ass $s_{R}\left(H_{I}^{t}(M, N)\right)$ is a finite set.

Proof. Apply 2.6 and the fact that the class of minimax modules includes all Noetherian and Artinian modules.

Corollary 2.8 The following statement are equivalent:

(i) $H_{I}^{0}\left(M, \Gamma_{I}(N)\right)$ is I-cofinite;

(ii) If $H_{I}^{i}(M, N)$ is minimax, for all $i<t$, then $\operatorname{Hom}_{R}\left(R / I, H_{I}^{t}(M, N) / K\right)$ is a finitely generated $R$-module, for any minimax submodule $K$ of $H_{I}^{i}(M, N)$. In particular, the associated primes of $H_{I}^{t}(M, N) / K$ are finite.

Proof. The proof, which we include for the reader's convenience, is based on Bahmanpour and Naghipour (2008 Theorem 2.4). It is enough to prove that $(i \Rightarrow i i)$. In view of the 2.6 the $R$-module $\operatorname{Hom}_{R}\left(R / I, H_{I}^{t}(M, N)\right)$ is finitely generated. On the other hand, according to Melkersson (2005 Proposition 4.3) $K$ is $I$-cofinite. Now, the exact sequence

$$
0 \longrightarrow K \longrightarrow H_{I}^{t}(M, N) \longrightarrow H_{I}^{t}(M, N) / K \longrightarrow 0
$$

induces the following exact sequence

$$
\operatorname{Hom}_{R}\left(R / I, H_{I}^{t}(M, N)\right) \longrightarrow \operatorname{Hom}_{R}\left(R / I, H_{I}^{t}(M, N) / K\right) \longrightarrow \operatorname{Ext}_{R}^{1}(R / I, K)
$$

consequently $\operatorname{Hom}_{R}\left(R / I, H_{I}^{t}(M, N) / K\right)$ is finitely generated.

\section{References}

[1] J. Asadollahi, K. Khashyarmanesh and Sh. Salarian, On the finiteness properties of the generalized local cohomology modules, comm. Algebra 30 (2002), 859-867. 
[2] K. Bahmanpour and R. Naghipour, On the cofiniteness of local cohomology modules, Proc. Amer. Math. Soc. 136 (2008), 2359-2363

[3] K. Borna, P. Sahandi and S. Yassemi, Cofiniteness of generalized local cohomology modules, Bull. Aust. Math. Soc. 83 (2011), 382-388.

[4] M. P. Brodmann and A. Lashgari Faghani, A finiteness result for associated primes of local cohomology modules, Proc. Amer. Math. Soc. 128 (2000), 2851-2853.

[5] K. Divaani-Aazar and M. A. Esmkhani, Artinianness of local cohomology modules of ZD-modules, Comm. Algebra 33 (2005), 2857-2863.

[6] J. Herzog, Auflosungen und dualitat in der lokalen algebra, Preprint univ. Regensburg, 1974.

[7] L. Melkersson, Modules cofinite with respect to an ideal, J. Algebra 285 (2005), 649-668.

[8] H. Saremi, On minimax and generalized local cohomology modules, Acta Mathematica Vietnamica 34 (2009), 269-273.

[9] M. Suzuki, On the generalized local cohomology and its duality, J. Math. Kyoto Univ. 18 (1978), 71-85.

[10] S. Yassemi, Generalized Section Functor, J. Pure and Applied Algebra 95(1994), 103-119.

[11] S. Yassemi, L. Khatami and T. Sharif, Associated primes of generalized local cohomology modules, Comm. Algebra 30 (2002), 327-330.

[12] H. Zöschinger, Minimax Moduln, J. Algebra 102 (1986), 1-32.

[13] H. Zöschinger, Über die Maximalbedingung für radikalvolle Untermoduln, Hokkaido Math. J. 17 (1988), 101-116.

Received: February 27, 2013 